\title{
Quasi-periodic solutions of the Boomeron equation
}

\author{
R Martini and W Wesselius \\ Department of Applied Mathematics, Twente University of Technology, PO Box 217, 7500 \\ AE Enschede, The Netherlands
}

Received 15 November 1984

\begin{abstract}
The quasi-periodic solutions for the Boomeron equation are determined by means of function-theoretical methods related to Riemann surfaces and $\theta$ functions. Also determined are the so-called Boomerons as degenerations of the quasi-periodic solutions. Moreover it is indicated that there are no higher-order Boomeron equations than the second-order one.
\end{abstract}

\section{Introduction}

The Boomeron equation was introduced not so very long ago by Calogero and Degasperis and shown to be solvable through the inverse scattering technique associated with the matrix Schrödinger equation $[2,3,4]$. In the present paper we report on quasi-periodic solutions of the Boomeron equation. Recently, several methods have been developed to obtain exact quasi-periodic solutions of nonlinear partial differential equations using function theoretical constructions related to Riemann surfaces and theta functions (particularly by Russian mathematicians $[5,6,9]$ ). These constructions involve Lax representations of the differential equations to be investigated. In [12] it was shown that the Boomeron equation admits such a Lax representation. Here we will apply the method explained with conspicuous clarity by Dubrovin [5] and thus obtain some results about quasi-periodic solutions of the Boomeron equation.

\section{The Boomeron equation}

For our convenience the Boomeron equation is written in the form

$$
W_{t x}=\left[\alpha, W_{x}\right]+\left\{W_{x x}, \beta\right\}+\left[W_{x},[W, \beta]\right]
$$

where $\alpha, \beta$ are given $2 \times 2$ matrices. $W$ is the unknown $2 \times 2$ matrix depending on $x$ and $t$ and the subscripts indicate partial differentiation. $[A, B]$ and $\{A, B\}$ denote the commutator and anticommutator of the matrices $A$ and $B$ respectively.

We define operators $L_{1}$ and $L_{2}$ by

$$
L_{1}=\mathrm{D}^{2}+W_{x}, \quad L_{2}=2 \beta \mathrm{D}+\alpha+[\beta, W]
$$

where $\mathrm{D}$ denotes the differential operator with respect to $x$. Then it can easily be 
shown that the Boomeron equation may be written in the form

$$
L_{1, t}=\left[L_{2}, L_{1}\right]
$$

a Lax representation (see [12]).

Usually, the unknown matrix $W, \alpha$ and $\beta$ are assumed to be Hermitian. In this case it is no restriction to suppose the matrix $\beta$ to be a real diagonal matrix. This is easily seen as follows. Since $\beta$ is Hermitian there exists a unitary matrix $P$ such that $P^{-1} \beta P=\tilde{\beta}$ is real and diagonal. For the operators $L_{1}$ and $L_{2}$ we can write after some computation

$$
L_{1}=P\left(D^{2}+\tilde{W}\right) P^{-1}=P\left(\tilde{L_{1}}\right) P^{-1}, \quad L_{2}=P(2 \tilde{\beta} D+\tilde{\alpha}+[\tilde{\beta}, \tilde{W}]) P^{-1}=P \tilde{L}_{2} P^{-1}
$$

with $\tilde{W}=P^{-1} W P$ and $\tilde{\alpha}=P^{-1} \alpha P$ and the Boomeron equation in the Lax form

$$
L_{1, t}=\left[L_{2}, L_{1}\right]
$$

becomes

$$
\tilde{L}_{1, t}=\left[\tilde{L}_{2}, \tilde{L}_{1}\right]
$$

Thus by the transformation $\tilde{W}=P^{-1} W P$ equation $(2.1)$ is reduced to the case in which $B$ is real and diagonal.

Calogero and Degasperis obtained equation (2.1) by a technique based on generalised Wronskian relations and usually write this equation in a different but equivalent form.

\section{Determination of the Baker-Akhiezer function}

We consider again the equation $\left[L_{2}, L_{1}\right]=L_{1, t}$, where $L_{1}=\sigma_{0} \mathrm{D}^{2}+W_{x}, L_{2}=$ $2 \beta \mathrm{D}+\alpha+[\beta, W]$ are operators in $\mathrm{D}$ with $2 \times 2$ matrices as coefficients. To get a better insight we introduce here the well known Pauli matrices:

$\sigma_{0}=\left[\begin{array}{ll}1 & 0 \\ 0 & 1\end{array}\right], \quad \sigma_{1}=\left[\begin{array}{ll}0 & 1 \\ 1 & 0\end{array}\right], \quad \sigma_{2}=\left[\begin{array}{cc}0 & -\mathrm{i} \\ \mathrm{i} & 0\end{array}\right], \quad \sigma_{3}=\left[\begin{array}{rr}1 & 0 \\ 0 & -1\end{array}\right]$

(with $\sigma_{i}^{2}=\sigma_{0}$ for $i=0,1,2,3$ ).

First we put the diagonal matrix $\beta$ in the form:

$$
\beta=\left[\begin{array}{cc}
\beta_{+} & 0 \\
0 & \beta_{-}
\end{array}\right]=\beta_{0}\left[\begin{array}{ll}
1 & 0 \\
0 & 1
\end{array}\right]+\beta_{3}\left[\begin{array}{rr}
1 & 0 \\
0 & -1
\end{array}\right]=\beta_{0} \sigma_{0}+\beta_{3} \sigma_{3}
$$

which means: $\beta_{ \pm}=\beta_{0} \pm \beta_{3}$ and $2 \beta_{0}=\beta_{+}+\beta_{-}, 2 \beta_{3}=\beta_{+}-\beta_{-}$. Also

$$
\alpha=\sum_{i=0}^{3} \alpha_{i} \sigma_{i}, \quad W=\sum_{i=0}^{3} W_{i} \sigma_{i}
$$

and

$$
\begin{aligned}
\alpha+[\beta, W] & =\alpha+2 \mathrm{i} \beta_{3}\left(W_{1} \sigma_{2}-W_{2} \sigma_{1}\right) \\
& =\alpha_{0} \sigma_{0}+\alpha_{3} \sigma_{3}+2 \mathrm{i} \beta_{3}\left(\alpha_{1}-W_{2}\right) \sigma_{1}+2 \mathrm{i} \beta_{3}\left(\alpha_{2}+W_{1}\right) \sigma_{2}
\end{aligned}
$$


As a consequence we have

$$
\begin{aligned}
L_{1}=U_{2} D^{2}+U_{0} \quad \text { with } U_{2} & =\sigma_{0}, \quad U_{0}=\sum_{i=0}^{3} W_{i, x} \sigma_{i} \\
L_{2}=V_{1} D+V_{0} \quad \text { with } V_{1} & =2 \beta_{0} \sigma_{0}+2 \beta_{3} \sigma_{3} \\
V_{0} & =\alpha_{0} \sigma_{0}+\alpha_{3} \sigma_{3}+2 \mathrm{i} \beta_{3}\left(\alpha_{1}-W_{2}\right) \sigma_{1}+2 \mathrm{i} \beta_{3}\left(\alpha_{2}+W_{1}\right) \sigma_{2} .
\end{aligned}
$$

Before continuing the procedure to find exact solutions of the Boomeron equation we observe that a central role is played by the concept of the Baker-Akhiezer (BA) function. Therefore we first give a survey of the theory about the BA function, which we adopt from reference [5].

We consider the nonlinear partial differential equations for the coefficients of the operators:

$$
L_{1}=\sum_{\alpha=0}^{n} U_{\alpha}(x, y, t) D^{\alpha}, \quad L_{2}=\sum_{\beta=0}^{m} V_{\beta}(x, y, t) D^{\beta},
$$

which are equivalent to the operator equation:

$$
\left[L_{1}-\partial / \partial y, L_{2}-\partial / \partial t\right]=0 \Leftrightarrow\left[L_{1}, L_{2}\right]=L_{2, y}-L_{1, t}
$$

In general (3.1) has matrix coefficients $U_{\alpha}^{i j}(x), V_{\beta}^{i j}(x), 1 \leqslant i, j \leqslant l$. In our case $n=2$, $m=1, l=2$.

Lemma. (Chapter 3, theorem 3.1.1 in [5]). For each regular Riemann surface $\Gamma$ of genus $g$ with a fixed point $Q$ and a non-special divisor of degree $g$ (we suppose a set of $g$ points $P_{1}, \ldots, P_{g}$ in general position) there exists a unique function (the so-called BA function) $\Psi(x, y, t ; P), P \in \Gamma$ with the following properties:

(1) Except at $Q, \Psi$ is meromorphic with poles in $P_{1}, \ldots, P_{g}$

(2) In a neighbourhood of $Q, \Psi$ can be represented in the form

$$
\Psi(x, y, t ; P)=\left(\sum_{s=0}^{\infty} \xi_{s}(x, y, t) k^{-s}\right) \exp (k x+Q(k) y+R(k) t)
$$

with $\xi_{0}=\sigma_{0}$ (as a normalisation condition for $\Psi$ ), $k^{-1}=k^{-1}(P)$ is a fixed local parameter, $k^{-1}(Q)=0$ and $Q(k)=q_{n} k^{n}+\ldots+q_{0}$ and $R(k)=r_{m} k^{m}+\ldots+r_{0}$ are polynomials with matrix coefficients.

For $\Gamma$ it is useful to choose the hyperelliptic Riemann surface $w^{2}=\prod_{i=1}^{2 p+1}\left(z-z_{i}\right)$ (without multiple roots).

Theorem. (theorem 3.1.2 in [5]). Let $\Psi=\Psi(x, y, t ; P)$ be a BA function constructed from $k x+Q(k) y+R(k) t$ and corresponding to some non-special divisor $D$ of degree g. Then $\Psi$ is a common solution of the two equations $\partial \Psi / \partial y=L_{1} \Psi ; \partial \Psi / \partial t=L_{2} \Psi$.

To determine $\Psi$ the following lemma is very useful.

Lemma. (Lemma 3.1.1 in [5]). $\Psi$ fulfills the asymptotic conditions:

$$
\begin{aligned}
& \left(L_{1}-\partial / \partial y\right) \Psi=\mathrm{O}\left(k^{-1}\right) \exp (k x+Q(k) y+R(k) t) \\
& \left(L_{2}-\partial / \partial t\right) \Psi=\mathrm{O}\left(k^{-1}\right) \exp (k x+Q(k) y+R(k) t) .
\end{aligned}
$$


In our case $Q(k)=k^{2} \sigma_{0}, R(k)=2 \beta k+\alpha$ appears as the right choice. So we can calculate $\xi_{1}$ from the condition that the coefficients of $k^{n} \exp (k x+Q(k) y+R(k) t)$ are zero for $n=2,1,0$.

The determination of $\xi_{1}$ is sufficient for the results of the next section.

To express $\xi_{1}$ we take for $\Psi$ the expression

$$
\left(\sum_{s=0}^{\infty} \xi_{s} k^{-s}\right) \exp \left[\left(k x+k^{2} y\right) \sigma_{0}+(2 \beta k+\alpha) t\right]
$$

and we shall abbreviate the exponential power in $\Psi$ as $\mathrm{e}^{\cdots}$. We consider

$$
\begin{aligned}
\left(L_{1}-\partial / \partial y\right) \Psi & =\left[\left(\sigma_{0} D^{2}+W_{x}\right)-\partial / \partial y\right]\left(\sum_{0}^{\infty} \xi_{s} k^{-s}\right) \mathrm{e}^{\cdots} \\
= & \left(\sum_{1}^{\infty} \xi_{s, x x} k^{-s}\right) \mathrm{e}^{\cdots}+2 k\left(\sum_{1}^{\infty} \xi_{s, x} k^{-s}\right) \mathrm{e}^{\cdots}+k^{2}\left(\sum_{0}^{\infty} \xi_{s} k^{-s}\right) \mathrm{e}^{\cdots} \\
& +W_{x}\left(\sum_{0}^{\infty} \xi_{s} k^{-s}\right) \mathrm{e}^{\cdots}-\left(\sum_{1}^{\infty} \xi_{s, y} k^{-s}\right) \mathrm{e}^{\cdots}-k^{2}\left(\sum_{0}^{\infty} \xi_{s} k^{-s}\right) \mathrm{e}^{\cdots}=0 .
\end{aligned}
$$

We multiply from the right by $\mathrm{e}^{-(\cdots)}$ and calculate the new expression modulo $\mathrm{O}\left(k^{-1}\right)$ and observe that the terms with factor $k^{2}, k$ cancel. The terms with $k^{0}$ deliver: $\xi_{0, x x}+2 \xi_{1, x}+W_{x}=0$. Since $\xi_{0}=\sigma_{0}$, we obtain

$$
2 \xi_{1, x}+W_{x}=0 \Rightarrow \xi_{i}=-\frac{1}{2} W+C(y, t ; P) .
$$

Moreover we consider

$$
\left(L_{2}-\partial / \partial t\right) \Psi=\left[2 \beta D+\alpha+2 \mathrm{i} \beta_{3}\left(W_{1} \sigma_{2}-W_{2} \sigma_{1}\right)-\partial / \partial t\right]\left(\sum_{0}^{\infty} \xi_{s} k^{-s}\right) \mathrm{e}^{\cdots}=0 .
$$

Hence

$$
\begin{aligned}
2 \beta\left(\sum_{1}^{\infty} \xi_{s, x} k^{-s}\right) & \mathrm{e}^{\cdots}+2 k \beta\left(\sum_{0}^{\infty} \xi_{s} k^{-s}\right) \mathrm{e}^{\cdots}+\left[\alpha+2 \mathrm{i} \beta_{3}\left(W_{1} \sigma_{2}-W_{2} \sigma_{1}\right)\right]\left(\sum_{0}^{\infty} \xi_{s} k^{-s}\right) \mathrm{e}^{\cdots} \\
& -\left(\sum_{1}^{\infty} \xi_{s, t} k^{-s}\right) \mathrm{e}^{\cdots}-\left(\sum_{0}^{\infty} \xi_{s} k^{-s}\right)(2 \beta k+\alpha) \mathrm{e}^{\cdots}=0 .
\end{aligned}
$$

Again we multiply from the right by $\mathrm{e}^{-(\cdots)}$ and calculate the expression modulo $\mathrm{O}\left(k^{-1}\right)$. Comparing the coefficients of $k$, and also $k^{0}$, we obtain:

$$
2 \beta \sigma_{0}-\sigma_{0} 2 \beta=0
$$

and

$$
2 \beta \xi_{1}-2 \xi_{1} \beta+\alpha \xi_{0}-\xi_{0} \alpha+\mathrm{i} 2 \beta_{3}\left[\begin{array}{cc}
0 & -W_{2}-\mathrm{i} W_{1} \\
-W_{2}+\mathrm{i} W_{1} & 0
\end{array}\right]=0 .
$$

The last expression can be reduced to

or to

$$
\left[\beta, \xi_{1}\right]=-\mathrm{i} \beta_{3}\left[\begin{array}{cc}
0 & -W_{2}-\mathrm{i} W_{1} \\
-W_{2}+\mathrm{i} W_{1} & 0
\end{array}\right]
$$

$$
2 \beta_{3}\left[\begin{array}{cc}
0 & \xi_{1}^{12} \\
-\xi_{1}^{21} & 0
\end{array}\right]=-\mathrm{i} \beta_{3}\left[\begin{array}{cc}
0 & -W_{2}-\mathrm{i} W_{1} \\
-W_{2}+\mathrm{i} W_{1} & 0
\end{array}\right]
$$


We distinguish two cases:

$$
\begin{aligned}
& \beta_{3}=0 \Rightarrow \beta_{+}=\beta_{-} \quad \text { (this case is trivial) } \\
& \beta_{3} \neq 0 \Rightarrow \beta_{+} \neq \beta_{-} .
\end{aligned}
$$

We continue with the last case and finally find

$$
\xi_{1}^{12}=\frac{1}{2}\left(-W_{1}+\mathrm{i} W_{2}\right), \quad \xi_{1}^{21}=-\frac{1}{2}\left(W_{1}+\mathrm{i} W_{2}\right) .
$$

Combining this result with (3.2) we obtain

$$
\xi_{1}=-\frac{1}{2}\left[\begin{array}{cc}
W_{0}+W_{3}+C_{11} & W_{1}-\mathrm{i} W_{2} \\
W_{1}+\mathrm{i} W_{2} & W_{0}-W_{3}+C_{22}
\end{array}\right]
$$

where $C_{11}$ and $C_{22}$ are constants with respect to $x$.

Remark. That the coefficients of $k^{2}, k$ in the first situation and the coefficient of $k$ in the second situation are fulfilled identically is a consequence of the right choice for $Q(k)$ and $R(k)$ in the BA function.

\section{Quasi-periodic solutions}

The purpose of this section is to find an explicit expression for $W(x, y, t)$. We start with some general observations in connection with $\theta$-functions. Let $\Gamma$ be a hyperelliptic Riemann surface with a branch point in $Q$. On the regular Riemann surface $\Gamma$ of genus $g$ we fix a basis of cycles $a_{1}, \ldots, a_{\mathrm{g}}, b_{1}, \ldots, b_{\mathrm{g}}$ with the intersection matrix $a_{k} \circ a_{j}=$ $b_{k} \circ b_{j}=0, a_{k} \circ b_{j}=\delta_{k j}(k, j=1, \ldots, g)$.

We introduce a basis of holomorphic differentials $\omega_{j}$ on $\Gamma$, normalised by $\oint_{a_{k}} \omega_{j}=$ $2 \pi \mathrm{i} \delta_{k j}$. We denote by $B$ the matrix of the $b$-periods $B_{k j}=\oint_{b_{k}} \omega_{j}$. This matrix is known to be symmetric and to have a positive definite imaginary part. The linear combinations (with coefficients in $\mathbb{Z}$ ) of vectors in $\mathbb{C}^{g}$ with coordinates $2 \pi \mathrm{i} \delta_{j k}$ and $B_{j k}$ form a lattice, which determines the complex torus $J(\Gamma)$, the so-called Jacobian variety of the Riemann surface. We have now the Abel-mapping $A: \Gamma \rightarrow J(\Gamma)$ with: $A(P)=\int_{P_{0}}^{P} \omega_{k}(k=1, \ldots, g$, $P_{0}$ is fixed point on $\Gamma$ ). From $B$ we can construct the Riemann $\theta$ function, that is an entire function with $g$ complex variables:

$$
\theta\left(z_{1}, \ldots, z_{g}\right)=\sum_{n \in \mathbf{Z}^{g}} \exp \left[\frac{1}{2}(B n, n)+(n, z)\right]
$$

where $(n, z)=\sum_{k=1}^{g} n_{k} z_{k}$ and $(B n, n)=\sum_{j, k=1}^{g} B_{j k} n_{j} n_{k}$.

This $\theta$ function has the following properties

$$
\begin{aligned}
& \theta\left(z_{1}, \ldots, z_{j}+2 \pi \mathrm{i}, \ldots, z_{g}\right)=\theta\left(z_{1}, \ldots, z_{j}, \ldots, z_{g}\right) \\
& \theta\left(z_{1}+B_{1 k}, \ldots, z_{g}+B_{g k}\right)=\exp \left(-\frac{1}{2} B_{k k}-z_{k}\right) \theta\left(z_{1}, \ldots, z_{g}\right) .
\end{aligned}
$$

In addition, for any non-special divisor $D=\sum_{j=1}^{g} P_{j}$ of degree $g$ there is a vector $A(D)$ such that the function $\theta(A(P)-A(D))$ defined on $\Gamma$, cut along the cycles $a_{k}, b_{j}$, has exactly $g$ zeros, which coincide with the $P_{j}$. If we denote by $\omega_{2}, \omega_{Q}$ and $\omega_{R}$, the normalised Abelian differentials of the second kind, that have a unique singularity at $Q$, are of the form $-\mathrm{d} z / z^{2}, \mathrm{~d} Q(1 / z), \mathrm{d} R(1 / z)$ in the local parameter $z(P)$.

Let $U_{1}, U_{2}, U_{3}$ be the vectors of their $b$-periods. 
From (4.2) it follows that

$\exp \left(\int_{P_{0}}^{P}\left(x \omega_{2}+y \omega_{Q}+t \omega_{R}\right)\right) \frac{\theta\left(A(P)+U_{1} x+U_{2} y+U_{3} t-A(D)-K\right)}{\theta(A(P)-A(D)-K)}$

does not change its value in a circuit around the cycles $a_{k}$ and $b_{j}$ and is therefore well defined. Normalising it in $P_{0}$ we obtain $\Psi(x, y, t ; P)$. The vector $K$ in the expression above is the vector of Riemann constants $\left(K_{1}, \ldots, K_{g}\right)$ and is connected in a simple way with the canonical class $C$ of $\Gamma: K=-\frac{1}{2} A(C)$ (see [5], p 37).

We continue our solution to establish exact solutions of the Boomeron equation. To simplify the problem for the moment we assume that in $\alpha=\Sigma_{i=0}^{3} \alpha_{i} \sigma_{i} \alpha_{1}=\alpha_{2}=0$, that means $\alpha$ is a diagonal matrix:

$$
\alpha=\left[\begin{array}{cc}
\alpha_{0}+\alpha_{3} & 0 \\
0 & \alpha_{0}-\alpha_{3}
\end{array}\right]
$$

We recall that

$$
\beta=\left[\begin{array}{cc}
\beta_{0}+\beta_{3} & 0 \\
0 & \beta_{0}-\beta_{3}
\end{array}\right]
$$

First we consider the exponential factor of the BA function.

$\Phi=\exp \left(k x+k^{2} y+2 \beta t k+\alpha t\right)$. As the matrices $\alpha$ and $\beta$ are diagonal we can put this exponential in the form (as a matrix):

$$
\Phi=\left[\begin{array}{cc}
\exp \left[k\left(x+2 \beta_{+} t\right)+k^{2} y+\left(\alpha_{0}+\alpha_{3}\right) t\right] & 0 \\
0 & \exp \left[k\left(x+2 \beta_{-} t\right)+k^{2} y+\left(\alpha_{0}-\alpha_{3}\right) t\right]
\end{array}\right]
$$

We introduce $\varepsilon_{1}=2 \beta_{+}, \varepsilon_{2}=2 \beta_{-}$.

In connection with (4.3) we have now found:

$$
\begin{array}{r}
\Psi^{i i}=\exp \left[\left(x+\varepsilon_{i} t\right) \int_{P_{0}}^{P} \Omega^{(1)}+y \int_{P_{0}}^{P} \Omega^{(2)}+t\left(\alpha_{0} \pm \alpha_{3}\right)\right] \\
\times \frac{\theta\left\{A(P)-A(D)-K+\left(x+\varepsilon_{i} t\right) U+y V\right\}}{\theta[A(P)-A(D)-K]}
\end{array}
$$

$\Psi^{i j}=0$ if $i \neq j \quad\left(\right.$ take $\alpha_{0}+\alpha_{3}$ if $i=1, \alpha_{0}-\alpha_{3}$ if $\left.i=2\right)$.

As $W_{x}=-2 \xi_{1, x}($ see $\S 3)$ and

$$
\xi_{1}^{i i}=-(\partial / \partial x) \log \theta\left\{-A(D)-K+\left(x+\varepsilon_{i} t\right) U+y V\right\}
$$

we obtain

$$
W^{i j}=2 \delta^{i j}(\partial / \partial x) \log \theta\left\{-A(D)-K+\left(x+\varepsilon_{i} t\right) U+y V\right\}+C,
$$

where we can consider $-A(D)-K$ as a constant $z_{0}$.

Proof. From (4.4) it follows (we abbreviate $\eta_{i}=\alpha_{0} \pm \alpha_{3}, i=1$ corresponds with + , $i=2$ with - )

$$
\begin{gathered}
\log \Psi^{i i}=\left(x+\varepsilon_{i} t\right) k+y k^{2}+t \eta_{i}+\log \theta\left\{A(P)-A(D)-K+\left(x+\varepsilon_{i} t\right) U+y V\right\} \\
-\log \theta\{A(P)-A(D)-K\}
\end{gathered}
$$


For $P \rightarrow Q$ we have:

Also

$$
A(P)=A(Q)-k^{-1} U+\mathrm{O}\left(k^{-2}\right) .
$$

$\log \theta\left\{A(P)-A(D)-K+\left(x+\varepsilon_{i} t\right) U+y V\right\}$

$$
=\log \theta\left\{-A(D)-K+\left(x+\varepsilon_{i} t\right) U+y V\right\}+\partial_{x} \log \theta\{\ldots\}-k^{-1} U_{j}+\mathrm{O}\left(k^{-2}\right)
$$

where $\{\ldots\}$ means: $-A(D)-K+\left(x+\varepsilon_{i} t\right) U+y V$.

From this it follows that

$$
W_{x}=-2 \xi_{1, x}=2 \frac{\partial}{\partial x} \sum_{j}\left(\frac{\partial_{j} \theta}{\theta}\{\ldots\} U_{j}\right)=2 \frac{\partial^{2}}{\partial x^{2}} \log \theta\{\ldots\} .
$$

We can split $\Psi^{i i}(x, y, t ; P)$ as follows:

$\Psi(x, y, t ; P)=\exp \left\{k x+k^{2} y+(2 \beta k+\alpha) t\right\}=\left(\exp k^{2} y\right) \exp \{k x+(2 \beta k+\alpha) t\}$.

We see $\phi(x, t ; P)=\exp \{k(x+2 \beta t)+\alpha t\}$ is the BA function with the same divisor of poles as $\Psi$ and with an essential singularity $\phi$ of the form $\exp \{k(x+2 \beta t)+\alpha t\}$ at $Q$. (This follows from the uniqueness of the BA function.)

So our problem is simplified to:

$$
\begin{aligned}
& L_{1} \phi(x, t ; P)=k^{2} \phi(x, t ; P) \\
& L_{2} \phi=\partial \phi / \partial t .
\end{aligned}
$$

The first of these equations (4.6) says that $\phi$ is an eigenfunction of the Sturm-Liouville operator $L_{1}$ with eigenvalue $k^{2}$ depending on $t$ by the second equation (4.7). The coefficients of $L_{1}$ and $L_{2}$ are now independent of $y$ and we receive as an exact solution of the Boomeron equation:

$$
W^{i j}=2 \delta^{i j}(\partial / \partial x) \log \theta\left\{z_{0}+\left(x+\varepsilon_{i} t\right) U\right\}+C .
$$

( $z_{0}, U$ are constants, $C$ is a diagonal matrix, constant WRT $x$.)

Remark. We considered above the case that $\alpha$ is a diagonal matrix. It is not difficult to reduce the general case with an arbitrary matrix $\alpha$ to the diagonal case. Suppose $\tilde{W}$ is a quasi-periodic (QP) solution of the general Boomeron equation (2.1). Now let $\gamma$ be the constant matrix such that

$$
[\gamma, \beta]=\alpha_{1} \sigma_{1}+\alpha_{2} \sigma_{2}
$$

It follows that

$$
\gamma=\gamma_{1} \sigma_{1}+\gamma_{2} \sigma_{2} \quad \text { with } \gamma_{1}=\mathrm{i} \alpha_{2} / 2 \beta_{3}, \gamma_{2}=-\mathrm{i} \alpha_{1} / 2 \beta_{3} .
$$

Now consider the matrix $W=\tilde{W}-\gamma$, then we have

$$
\begin{aligned}
W_{l x} & =\left[\alpha, W_{x}\right]+\left\{W_{x x}, \beta\right\}+\left[W_{x},[W+\gamma, \beta]\right] \\
& =\left[\alpha, W_{x}\right]+\left\{W_{x x}, \beta\right\}+\left[W_{x},[W, \beta]\right]+\left[W_{x}, \alpha_{1} \sigma_{1}+\alpha_{2} \sigma_{2}\right] \\
& =\left[\alpha_{0} \sigma_{0}+\alpha_{3} \sigma_{3}, W_{x}\right]+\left\{W_{x x}, \beta\right\}+\left[W_{x},[W, \beta]\right] .
\end{aligned}
$$

Then $W$ is a QP solution of the case $\alpha_{1}=\alpha_{2}=0$.

Conversely, if $W$ is a QP solution of the special case $\alpha_{1}=\alpha_{2}=0$ then $W+\gamma$, with $\gamma$ as in (4.9), is a QP solution of the general case. 
Finally in the general case the solution has the formula

$\tilde{W}^{i j}=2 \delta^{i j}(\partial / \partial x) \log \theta\left(z_{0}+\left(x+\varepsilon_{i} t\right) U\right)+C_{0} \sigma_{0}+\gamma_{1} \sigma_{1}+\gamma_{2} \sigma_{2}+C_{3} \sigma_{3}$

with arbitrary constants $C_{0}$ and $C_{3}$ with respect to $x$.

Example. We illustrate this result by choosing for $\Gamma$ the hyperelliptic surface $w^{2}=$ $4 z^{3}-g_{2} z-g_{3}$ of genus 1 . In this case we get ( $\theta_{1}$ is the one of the four basis $\theta$ functions in the case $g=1$; see [5] p 17):

$$
W_{x}^{i j}=2 \delta^{i j}\left(\partial^{2} / \partial x^{2}\right) \log \theta_{1}\left(z_{0}+\left(x+\varepsilon_{i} t\right) U\right)+C_{1} .
$$

The relation: $\mathscr{P}(z)=-\left(\partial^{2} / \partial z^{2}\right) \log \theta_{1}(z)+\eta$, where $\mathscr{P}(z)$ is the Weierstrasz $\mathscr{P}$-function and $\eta$ is a known constant is well known (see [14], pp 265, 286).

Hence

$$
W_{x}^{i j}=-2 \delta^{i j} \mathscr{P}\left\{z_{0}+\left(x+\varepsilon_{i} t\right) U\right\}+C .
$$

As $\zeta^{\prime}(z)=-\mathscr{P}(z)$, where $\zeta(z)$ is the Weierstrasz $\zeta$-function ([14], p 260) we obtain

$$
W_{x}^{i j}=2 \delta^{i j}(\partial \zeta / \partial x)\left\{z_{0}+\left(x+\varepsilon_{i} t\right) U\right\}+C
$$

and hence

$$
W^{i j}=2 U^{-1} \delta^{i j} \zeta\left\{z_{0}+\left(x+\varepsilon_{i} t\right) U\right\}+C(t) x+D(t)
$$

is an exact QP solution of (2.1).

( $\varepsilon_{1}=2 \beta_{0}+2 \beta_{3}, \varepsilon_{2}=2 \beta_{0}-2 \beta_{3}, C$ is a diagonal matrix and constant wRT $x, U, z_{0}$ are constants.)

\section{Degenerations and Boomerons}

Manin [11] observed that for the $\mathrm{KDV}$ equation $u_{t}-6 u u_{x}+u_{x x x}=0$ there is a solution in the form of a travelling wave $u(x, t)=U(x-v t)$. This solution is found by integrating

$$
\left(U^{\prime}\right)^{2}=2 U^{3}+v U^{2}+a U+b .
$$

The general solution is $U(x-v t)=c_{1} \mathscr{P}(x-v t)+c_{2}$ (where $\mathscr{P}$ is the Weierstrasz elliptic function). The periods of this function are the periods of the elliptic curve $\Gamma$

$$
w^{2}=2 z^{3}+v z^{2}+a z+b .
$$

This is a cnoidal wavetrain if the discriminant of the curve $\Delta=g_{2}^{3}-27 g_{3}^{2} \neq 0$ (see [7]). The period of the wavetrain is the real period of $\Gamma$, i.e. $\int_{\gamma} \omega$, where

$$
\omega=\mathrm{d} z\left(2 z^{3}+v z^{2}+a z+b\right)^{-1 / 2}
$$

and $\gamma$ is a real cycle on the Riemann surface of $\Gamma$. The soliton is obtained for the curve with a double point at the origin $w^{2}=2 z^{3}+v z^{2}, a=b=0$ (so $\Delta=0$ ) and the solution is

$$
U(x-v t)=-\frac{1}{2} v \cosh ^{-2}\left[\frac{1}{2} v^{1 / 2}(x-v t)\right] .
$$

The soliton is the limit of the cnoidal wavetrain when its period tends to infinity. We use now the same consideration to determine the Boomeron as degeneration of our general solutions in $\$ 4$. 
Consider the hyperelliptic surface

$$
w^{2}=4 a z^{3}+6 b z^{2}+4 c z+d .
$$

With this surface is associated the following well known function:

$$
\begin{aligned}
u=\int \frac{\mathrm{d} z}{w}=\int \frac{\mathrm{d} z}{\left(4 a z^{3}+6 b z^{2}+4 c z+d\right)^{1 / 2}}=\int \frac{\mathrm{d} x}{\left(4 x^{3}-g_{2} x-g_{3}\right)^{1 / 2}} \\
==\mathscr{P}\left(x ; g_{2}, g_{3}\right)=\mathscr{P}\left(a z+\frac{1}{2} b ; 3 b^{2}-4 a c, 2 a b c-b^{3}-a^{2} d\right)
\end{aligned}
$$

where we applied the substitution $z=\left(x-\frac{1}{2} b\right) / a$ and $\mathscr{P}$ denotes the Weierstrasz function. From [7] (p 339) we borrow the relations $\zeta^{\prime}(t)=-\mathscr{P}(t)$ ( $\zeta$ is the Weierstrasz $\zeta$ function) and in the case of degeneration we obtain ([7] p 339)

$$
\zeta_{0}(s)=-\frac{1}{2} b s+\left(\frac{3}{2} b\right)^{1 / 2} \operatorname{coth}\left\{\left(\frac{3}{2} b\right)^{1 / 2} s\right\}
$$

where $\zeta_{0}$ denotes the degenerated $\zeta$ function (in (1) $c=d=0$ ).

In $\S 4$ we found as solution:

$$
W^{i j}=2 U^{-1} \delta^{i j} \zeta\left\{z_{0}+\left(x+\varepsilon_{i} t\right) U\right\}+x C(t)+D(t)
$$

where $C(t)$ is a diagonal matrix, $U \neq 0, \varepsilon_{i}=2 \beta_{i}(i=1,2)$.

In our case we carry out the substitution $x=a X+\frac{1}{2} b$ and the result is (the subscript 0 again denotes the degenerated solution)

$$
\begin{aligned}
W_{0}^{i j}=2 U^{-1} \delta^{i j}[ & -\frac{1}{2} b\left\{z_{0}+\left(a X+\frac{1}{2} b+\varepsilon_{i} t\right) U\right\}+\left(\frac{3}{2} b\right)^{1 / 2} \operatorname{coth}\left[\left(\frac{3}{2} b\right)^{1 / 2}\left\{z_{0}+\left(a X+\frac{1}{2} b+\varepsilon_{i} t\right) U\right\}\right] \\
& +C(t)\left(a X+\frac{1}{2} b\right)+D(t) .
\end{aligned}
$$

We check this solution by substituting in

$$
W_{t x}=\left[\alpha, W_{x}\right]+\left\{W_{x x}, \beta\right\}-\left[W_{x},[W, \beta]\right] \quad \text { with } \alpha=\left[\begin{array}{cc}
\alpha_{1} & 0 \\
0 & \alpha_{4}
\end{array}\right] \beta=\left[\begin{array}{cc}
\beta_{1} & 0 \\
0 & \beta_{4}
\end{array}\right] \text {. }
$$

Completely written out the equation is

$$
\begin{array}{lc}
W_{t x}^{1}= & 2 \beta_{1} W_{x x}^{1}+\left(\beta_{1}-\beta_{4}\right)\left(W_{2} W_{3}\right)_{x} \\
W_{t x}^{2}= & \left(\alpha_{1}-\alpha_{4}\right) W_{x}^{2}+\left(\beta_{1}+\beta_{4}\right) W_{x x}^{2}+\left(\beta_{1}-\beta_{4}\right)\left(W_{4}-W_{1}\right)_{x} W_{2} \\
W_{t x}^{3}= & -\left(\alpha_{1}-\alpha_{4}\right) W_{x}^{3}+\left(\beta_{1}+\beta_{4}\right) W_{x x}^{3}+\left(\beta_{1}-\beta_{4}\right)\left(W_{4}-W_{1}\right)_{x} W_{3} \\
W_{t x}^{4}= & 2 \beta_{4} W_{x x}^{4}-\left(\beta_{1}-\beta_{4}\right)\left(W_{2} W_{3}\right)_{x}
\end{array}
$$

(the superscripts denote the elements of the matrix $W$, the nonlinearity of the Boomeron equation is demonstrated in the last terms of the right-hand side). Substitution of (5.5) in (5.7) results: $a=1, \varepsilon_{i}=2 \beta_{i}, D$ is diagonal. This can be explained as follows

$a=1$ is not a special value, because of the factor $U$.

$\varepsilon_{i}=2 \beta_{i}$ was also found in our earlier results.

$D$ diagonal is a consequence of the choice of $\alpha$ and $\beta$ as diagonal matrices. The same solution was found by Calogero and Degasperis in [2] (p 429) and [4] (p 311, 312) in different ways. We can also obtain the so-called double degenerated case that means in (5.1) $b=0$ too.

From [7] (p 340) we then get

$$
W^{i j}=\frac{\delta^{i j}}{\left(a X+2 \beta_{i} t+\frac{1}{2} b\right) U}+C(t)\left(a X+\frac{1}{2} b\right)+D(t) \quad \begin{aligned}
& \text { with } a=1 \text { and } C, D \\
& \text { diagonal matrices. }
\end{aligned}
$$


Another approach to tackle the degenerated solutions of the Boomeron equation can be received by using the methods reported in McKean: global analysis [13] based on the work by Fay [8]. On pages 97,137 he mentioned the useful formula:

$\theta_{0}(x)=\sum_{n} e^{x . n} \prod_{i=j}\left(\frac{k_{1}-k_{j}}{k_{i}+k_{j}}\right)^{2 n_{1} \cdot n_{,}}, \quad$ where $x=\left(x_{1}, \ldots, x_{g}\right) \in \mathbb{C}^{g}$ is a $g$-vector,

$0<k_{1}<k_{2}<\ldots<k_{g}$ are fixed and the sum is taken over all $n=\left(n_{1}, \ldots, n_{g}\right)$ with $n_{t}=0$ or $1(i=1, \ldots, g)$.

If $g=1$ we have $\theta_{0}(x)=1+\mathrm{e}^{x_{1}}$.

So our solution takes the form:

$$
\begin{aligned}
W^{i j} & =2 \delta^{i j}(\partial / \partial x) \log \theta_{0}\left(z_{0}+\left(x+\varepsilon_{i} t\right) U\right)+C \\
& =2 \delta^{i j}(\partial / \partial x) \log \left(1+\exp \left(z_{0}+\left(x+\varepsilon_{i} t\right) U\right)+C\right. \\
& =2 \delta^{i j} U \mathrm{e}^{s_{1}} /\left(1+\mathrm{e}^{s_{2}}\right)+C, \quad \text { where } s_{i}=\left(x+\varepsilon_{i} t\right) U+z_{0} \\
& =\delta^{i j} U\left\{1-\tanh \left(-\frac{1}{2} s_{i}\right)\right\}+C
\end{aligned}
$$

(as was found in [2] and [4]).

If $g=2$ we get a more complicated result:

$$
\begin{aligned}
\theta_{0}(x) & =\sum_{n} \exp \left(x_{1} n_{1}+x_{2} n_{2}\right) \prod_{\substack{i<j \\
i=1,2 \\
j=1,2}}\left(\frac{k_{i}-k_{j}}{k_{i}+k_{j}}\right)^{2 n_{1} n_{j}} \quad\left(k_{i}<k_{j}\right) \\
& =1+\exp \left(x_{1}\right)+\exp \left(x_{2}\right)+\exp \left(x_{1}+x_{2}\right)\left(\frac{k_{1}-k_{2}}{k_{1}+k_{2}}\right)^{2}
\end{aligned}
$$

This substituted in (4.10) yields (abbreviating $s_{j}=z_{0 j}+\left(x+\varepsilon_{i} t\right) U_{j} ; j=1,2$ )

$$
\begin{aligned}
W^{i j}=2 \delta^{i j}(\partial / \partial x) \log \theta_{0}\left(z_{0}+\left(x+\varepsilon_{i} t\right) U\right)+C \\
\quad=2 \delta^{i j}(\partial / \partial x) \log \left[1+\exp \left(s_{1}\right)+\exp \left(s_{2}\right)+\exp \left(s_{1}+s_{2}\right)\left(\frac{k_{1}-k_{2}}{k_{1}+k_{2}}\right)^{2}\right] \\
=2 \delta^{i j} \frac{U_{1} \exp \left(s_{1}\right)+U_{2} \exp \left(s_{2}\right)+\lambda\left(U_{1}+U_{2}\right) \exp \left(s_{1}+s_{2}\right)}{1+\exp \left(s_{1}\right)+\exp \left(s_{2}\right)+\lambda \exp \left(s_{1}+s_{2}\right)}+C \\
=2 \delta^{i j} \frac{U_{1} \exp \left(-s_{2}\right)+U_{2} \exp \left(-s_{1}\right)+\lambda\left(U_{1}+U_{2}\right)}{\left[\exp \left(-s_{1}\right)+1\right]\left[\exp \left(-s_{2}\right)+1\right]+\lambda-1}+C, \quad \text { with } \lambda=\left(\frac{k_{1}-k_{2}}{k_{1}+k_{2}}\right)^{2}
\end{aligned}
$$

which is the same result as that found by Calogero and Degasperis ([4], pp 311, 313), namely the two-soliton case. If we introduce $t$-dependency then it is possible to obtain more general solutions.

Remark. It is easy to check by straightforward calculation that the solution found in (5.10) but with $\varepsilon_{i} t$ replaced by a nonlinear function $\xi(t)$ also satisfies (5.7). This corresponds to the results of $[4]$ (p 311).

\section{Higher-order Boomeron equation}

We observed in $\S 1$ that the Boomeron equation

$$
W_{x t}=\left[\alpha, W_{x}\right]+\left\{W_{x x}, \beta\right\}+\left[W_{x},[W, \beta]\right] \quad \text { in matrix form }
$$


can be put in the $L a x$ representation $L_{t}=[B, L]$ where $L=D^{2}+W_{x}$ and $B=$ $2 \beta D+\alpha+[\beta, W] . \quad B$ is a skew symmetric operator if $\beta$ is Hermitian and $\alpha$ is antiHermitian. Also we say that it is possible to put $\beta$ in diagonal form and also $\alpha$ (see remark in $\$ 4$ ).

In what follows we are only interested in general solutions for $W$. We shall now take for $B_{n}$, where the highest order of $D$ is $2 n+1$, a higher-order operator (for $L$ we take the same operator as above), skew symmetric, and call the operation which follows from $L_{t}=\left[B_{n}, L\right] n$th higher-order Boomeron equation [1]. To skip the complicated computations we only mention the results. The first higher-order operator we try is

$$
B_{1}=a D^{3}+b D+D b+c, \quad \text { where } a, b, c \text { are } 2 \times 2 \text { matrices. }
$$

After some tedious calculations we obtain

$$
a=4 \gamma \sigma_{0}(\gamma \in C), \quad b=3 \gamma W_{x}+b_{0}, \quad c=\left[b_{0}, w\right]+c_{0}
$$

where $b_{0}$ is symmetric and $c_{0}$ is skew symmetric constant matrices with respect to $x$. The corresponding first higher-order Boomeron equation has the form

$$
W_{x t}=\gamma W_{x x x x}+3 \gamma\left\{W_{x x}, W_{x}\right\}+\left[c_{0}, W_{x}\right]+\left\{W_{x x}, b_{0}\right\}+\left[W_{x},\left[W, b_{0}\right]\right]
$$

The next attempt is

$$
B_{1}=e D^{5}+a D^{3}+D^{3} a+b D+D b+c .
$$

The calculations are now even more complicated. They have been carried out in REDUCE, a system for symbolic computations.

The results in this case are

$$
\begin{aligned}
& e=16 \gamma \sigma_{0}(\lambda \in C), \quad a=20 \lambda W_{x}+2 \mu \sigma_{0}(\mu \in C), \\
& b=15 \lambda W_{x}^{2}-5 \lambda W_{x x x}+3 \mu W_{x}+b_{0} \quad\left(b_{0} \text { is symmetric }\right), \\
& c=5 \lambda\left[W_{x}, W_{x x}\right]+\left[b_{0}, W\right]+c_{0} \quad\left(c_{0} \text { is skew symmetric }\right)
\end{aligned}
$$

and for the second higher-order Boomeron equation we obtain

$$
\begin{gathered}
W_{x t}=\lambda W_{6 x}+5 \lambda\left\{W_{4 x}, W_{x}\right\}+10 \lambda\left\{W_{3 x}, W_{2 x}\right\}+10 \lambda\left\{W_{2 x}, W_{x}^{2}\right\}+10 \lambda W_{x} W_{x x} W_{x}+\mu W_{4 x} \\
\left.+\left\{W_{x x}, b_{0}\right\}+3 \mu\left\{W_{x x}, W_{x}\right\}+\left[c_{0}, W_{x}\right]+\left[W_{x}, b_{0}\right]\right] .
\end{gathered}
$$

If we try the next step, namely to investigate

$$
B_{3}=f D^{7}+e D^{5}+D^{5} e+a D^{3}+D^{3} a+b D+D b+c
$$

then the result of calculations yields $f=0$. The reason for this is that the representation $L_{t}=\left[B_{3}, L\right]$ imposes more conditions for $f$, so the higher-order Boomeron equations collapse. This means there are only three order types of Boomeron equations where we start from the Lax representation. Of course the highest (second) order contains the lower-order equations by specialising the principal coefficients. The fact that the chain of higher-order Boomeron equations breaks down is most surprising, because we know that using the same method for the KdV equation one gets a hierarchy of higher-order $\mathrm{KdV}$ equations (see [10]). 


\section{Conclusion}

We conclude this paper with some remarks. The same methods as described in $\S 6$ may be applied to somewhat more complicated situations. For example one can consider the same equation but with matrices $\alpha$ and $\beta$ depending on $y$ and $t$. Also if the equations have the same structure but with $l \times l$ matrices instead of $2 \times 2$ matrices the scheme will work. Finally we can consider Riemann surfaces $\Gamma$ of higher genera $(g>2)$ to get other QP solutions and also can determine their degenerations.

Note added in proof. The Seminar on theta functions and related topics held at the Centre of Mathematics and Computer Science, Amsterdam, contributed to the realisation of this paper.

\section{References}

[1] Brands A 1983 Report on Determination of Lax representations of higher order Boomeron equations Twente University, Enschede (in Dutch)

[2] Calogero F and Degasperis A 1976 Lett. Nuovo Cimento 16425

[3] Calogero F and Degasperis A 1976 Lett. Nuovo Cimento 16 434-8

[4] Calogero F and Degasperis A 1980 in Solitons, ed R K Bullough and F J Caudrey (Berlin: Springer)

[5] Dubrovin B A 1981 Russian Math. Surveys 36 2, 11-92

[6] Dubrovin B A, Matveev V B and Novikov S P 1976 Russian Math. Surveys 31 1, 59-146

[7] Erdélyi A 1953 Higher Transcendent Functions vol II

[8] Fay J 1973 Lecture Notes in Math. 352 (Berlin: Springer)

[9] Krichever I M 1977 Russian Math. Surveys 31 6, 185-213

[10] Lax P D 1968 Commun. Pure Appl. Math. 21 467-90

[11] Manin Y I 1979 J. Sov. Math. 11 1, 1-122

[12] Martini R 1983 Proc. Kon. Ned. Akad. Wetensch. A 86 221-7

[13] McKean H P 1978 Lecture Notes in Math. 755 (Berlin: Springer) pp 83-200

[14] Rühs F 1962 Funktionen Theorie (Berlin: VEB) 